Research Article

\title{
Galactosamine-Conjugating Zwitterionic Block Copolymer for Reduction-Responsive Release and Active Targeted Delivery of Doxorubicin to Hepatic Carcinoma Cells
}

\author{
Jingming Zhai, ${ }^{1}$ Biyu Zhou, ${ }^{2}$ Yanhui An, ${ }^{1}$ Binzhong Lu, ${ }^{2}$ Yonggang Fan $\mathbb{D},{ }^{1}$ and Junbo Li $\mathbb{D}{ }^{1,2}$ \\ ${ }^{1}$ The First Affiliated Hospital and College of Clinical Medicine, Henan University of Science and Technology, Luo Yang 471023, China \\ ${ }^{2}$ School of Chemical Engineering \& Pharmaceutics, Henan University of Science \& Technology, Luo Yang 471023, China
}

Correspondence should be addressed to Yonggang Fan; fanyonggang196809@163.com and Junbo Li; lijunbo@haust.edu.cn

Received 16 January 2020; Accepted 4 March 2020; Published 20 April 2020

Guest Editor: Anuj Kumar

Copyright ( 2020 Jingming Zhai et al. This is an open access article distributed under the Creative Commons Attribution License, which permits unrestricted use, distribution, and reproduction in any medium, provided the original work is properly cited.

\begin{abstract}
Nanocarriers with integrated advantage, such as excellent stealth property, active targeting function, and rapid intracellular drug release, are significant for cancer treatment. Herein, a biodegradable zwitterionic triblock copolymer containing disulfide-linked poly- $\varepsilon$-caprolactone and polycarboxybetaine methacrylate (PCB-SS-PCL-SS-PCB) was first synthesized and then partly modified with galactosamine (GAL) for constructing polymeric micelle drug carrier with multifunctionality. Polymeric micelles showed ultralow protein absorption in serum and obvious reduction-responsiveness in the presence of glutathione, provided by the zwitterionic polymer shell and the disulfide bond, respectively. Furthermore, active targeting of the carrier to hepatic carcinoma cells was achieved via GAL ligands on PCB shells due to their specific binding to asialoglycoprotein receptors on the cell surface. As expected, in vivo competition studies demonstrated that doxorubicin- (DOX-) loaded GAL-modified micelles have better anticancer effect in hepatic tumor-bearing mice than free DOX and nontargetable micelles. As a result, this novel multifunctional carrier provides a valuable strategy to design promising anticancer drug delivery systems for liver cancer treatment.
\end{abstract}

\section{Introduction}

Chemotherapeutics are still the main treatment strategy for human cancer [1]. To reduce side effects and enhance bioavailability of hydrophobic drugs, nanodrug delivery systems (NDDS) have been widely explored in cancer chemotherapeutics during past decades [2, 3]. Generally, therapeutic nanoparticles required a hydrophilic coating to ensure a stealthy and long circulation in the bloodstream [4]. To date, polyethylene glycol (PEG) is the most widely used biointerface materials to prevent nonspecific protein adhesion on nanoparticles $[5,6]$, resulting in a passive accumulation of cargo in the tumor site via a so-called enhanced permeability and retention (EPR) effect [7, 8]. However, recent reports revealed that anti-PEG immunoglobulin antibodies detected in PEGylated systems after repeated administration lead to an insufficient antitumor efficacy caused by an accelerated blood clearance (ABC) phenomenon $[9,10]$.
Zwitterionic polymers are also neutral and ultrahydrophilic since they bear an equimolar number opposite ionizable group in monomeric unit [1]. The unique structure enables a zwitterionic layer to tightly bind water molecules via ion interaction, giving rise in ultrahigh resistance to protein adsorption and excellent blood compatibility. Moreover, without detectable immune response and avoiding $\mathrm{ABC}$ phenomenon, zwitterionic polymers have been also identified as better biointerface material alternative PEG $[11,12]$. Recently, a variety of zwitterionic therapeutic nanoparticles, such as polymer-drug conjugates [13], nanogels [14], and polymeric micelles [15] have been developed. However, overcoming their less efficient tumor cell uptake is still a challenge [16].

Among zwitterionic polymers, such as polyphosphobetaine and polysulfobetaine, polycarboxybetaine (PCB) has superior abilities because of its reactive carboxylate anion, which can readily conjugate with amine-containing biomolecules via simple carbodiimide chemistry [17]. Although the 


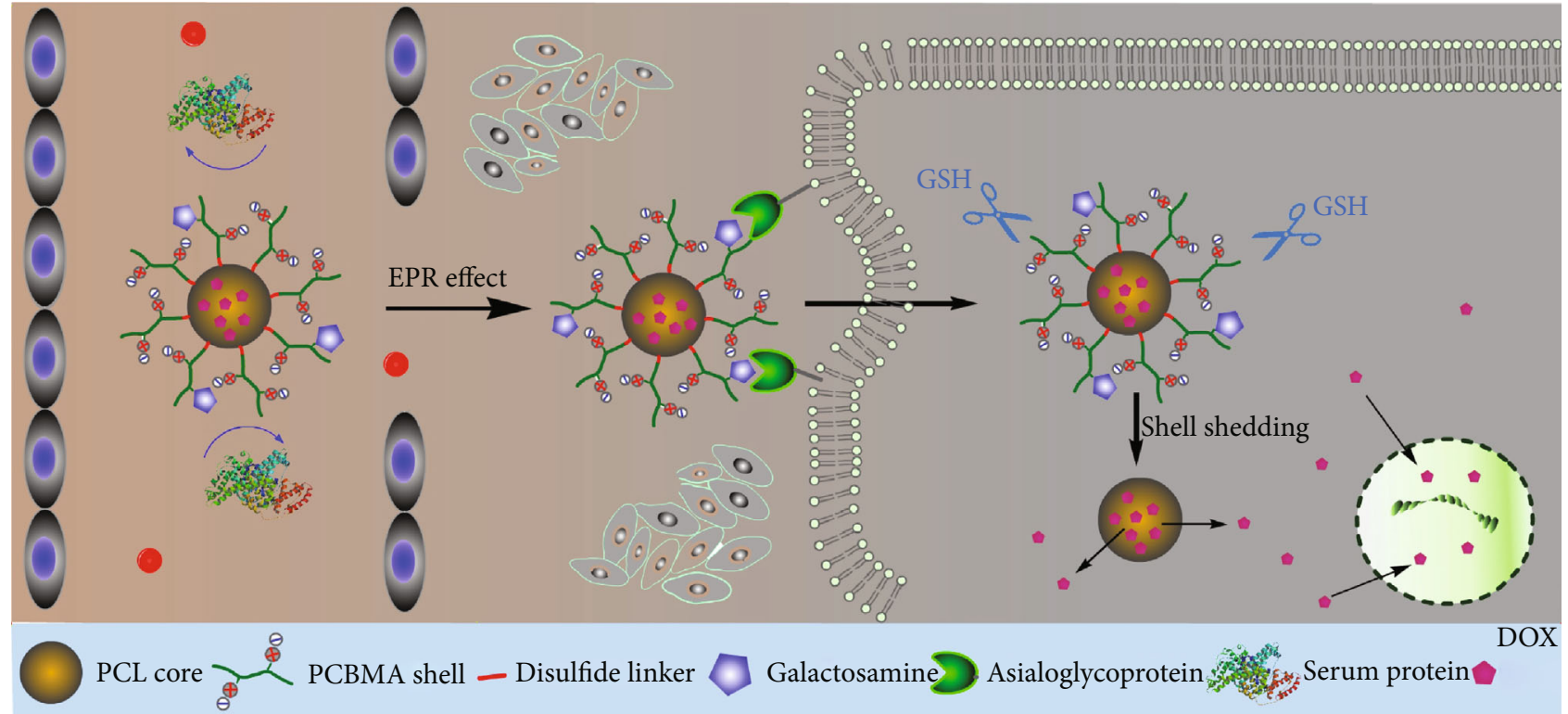

Scheme 1: Schematic representation of the structure of the multifunctional micelle carrier and its resistance protein adsorption in blood circulation, active targeting cell uptake to HepG2, and GSH-triggered intracellular drug release behavior.

modification has little influence on the antifouling property, it introduces novel functions [18-20]. For instance, to address the reduced cell uptake efficacy of zwitterionic nanoparticles, Lin et al. used c(RGDyK)-modified PCB to obtain micelle carriers with prolonged blood circulation time and active targeting property to improve therapeutic efficacy in vivo [21]. Furthermore, based on the specific recognition of asialoglycoprotein (ASGP) receptors to glycoproteins on hepatic carcinoma cells $[22,23]$, the immobilization of galactosamine on PCB substrates allows the adhesion-based selection of hepatocytes differing from other cell types [24]. Jiang's group also used fluorescein and galactose with the amine group to modify PLGA-PCB nanoparticles for selectively binding HepG2 cells [25]. The modification of galactose ligand on zwitterionic nanoparticles has exhibited an integrated ultrahigh antifouling and tumor-targeting properties to hepatic carcinoma cells. However, the in vivo antitumor effect of such NDDS needs further invistigation.

In addition to long circulation and tumor-targeting properties, an optimal micellar carrier should precisely control drug release inside the cancer cell. However, therapeutic efficacy is always restricted by the gradual drug permeation for its hydrophobicity [26]. The development of stimuli-responsive carriers that quickly release encapsulated cargo triggered by intracellular environmental factor is an efficient strategy to improve drug bioavailability [27-29]. In our recent paper, we developed micelle platform with a PCB shell and disulfide linked to a biodegradable poly- $\varepsilon$ caprolactone (PCL) core. The carriers showed many advantages, such as ultralow protein absorption in serum and a fast intracellular drug release triggered by higher GSH intracellular concentration [30]. Herein, to enhance drug bioavailability in vivo, this micellar carrier was further optimized by introduction of a galactosamine ligand for integrating the multifunction, excellent stealth property, active targeting to hepatic carcinoma, and reductively responsive intracellular release, as showing in Scheme 1.

\section{Materials and Methods}

2.1. Materials. PCL-diol $(\mathrm{Mn}=2000, \mathrm{PDI}=1.2)$ was purchased from Sigma-Aldrich (Shanghai, China). Cystamine dihydrochloride (Cys.2HCl) (>96\%) and 1, 1-carbonyldiimidazole (CDI) $(>97.0 \%)$ were purchased from the National Pharmaceutical Group Chemical Reagent (Beijing, China) and used as received. 2, 2-Azobis(isobutyronitrile) (AIBN) (97\%) was purchased from J\&K Chemical (Beijing, China) and recrystallized before being used as an initiator. The monomers, carboxybetaine methacrylate (CBMA, 99\%), RAFT reagent (4-cyanopentanoic acid)-4-dithiobenzoate (CPADB, $98 \%)$, doxorubicin hydrochloride (DOX.HCl, 99\%), N-(3dimethylaminopropyl)- $\mathrm{N}^{\prime}$-ethyl carbodiimide hydrochloride (EDC. $\mathrm{HCl}, 98 \%$ ), and N-hydroxysuccinimide (NHS, 98\%), were purchased from J\&K Chemical (Beijing, China) and used as received. All other reagents and solvents were of analytical grade and used as received. Dulbecco's modified Eagle's medium (DMEM), penicillin-streptomycin, fetal bovine serum (FBS), and 3-[4, 5-dimethylthiazol-2-yl]-2, 5-diphenyltetrazolium bromide (MTT), were purchased from Thermo Fisher Scientific (Shanghai, China).

2.2. Synthesis of PCL Macro-RAFT Agent. For synthesis of the PCL macro-RAFT agent, two hydroxyl groups of PCL-diol were first activated by CDI and then conjugated with cystamine monomer to provide reductive disulfide bonds and reactive amino-end groups [29]. Next, the PCL macroRAFT agent, CPADB-SS-PCL-SS-CPADB, was prepared by a condensation reaction between amino-end groups of $\mathrm{PCL}$ and the carboxylic group of CPADB through EDC/NHS chemistry. The detailed synthesis and characterization of 
the PCL macro-RAFT agent were described in our previous paper [28].

\subsection{Synthesis of PC-SS-PCL-SS-PCB. PCB-SS-PCL-SS-PCB} was prepared by RAFT polymerization of CBMA by using CPADB-SS-PCL-SS-CPADB as the chain transfer agent. Typically, The CPADB-SS-PCL-SS-CPADB $(0.4 \mathrm{~g}$, $0.140 \mathrm{mmol}$ ) and AIBN $(4.6 \mathrm{mg}, 0.028 \mathrm{mmol})$ were dissolved in a sealed ampoule with $4 \mathrm{ml}$ dry tetrahydrofuran (THF). Then, CBMA $(0.6 \mathrm{~g}, 2.618 \mathrm{mmol})$ was dissolved in $4 \mathrm{ml}$ saturated salt water and placed into an above reaction tube. After three freeze-evacuate-thaw cycles, the polymerization was conducted at $70^{\circ} \mathrm{C}$ for $48 \mathrm{~h}$. The product was purified by dialysis (bag MWCO 3000) against deionized water to remove salt and unreacted CBMA. Finally, PCB-SS-PCLSS-PCB was obtained by lyophilization as a light pink powder (0.574g, 57.4\%).

2.4. Modification of PCB-SS-PCL-SS-PCB with Galactosamine. Targeting molecule, GAL is used to conjugate on PCB-SSPCL-SS-PCB by an amidation reaction between the amine of GAL and carboxylate group of PCB. To accomplish the purpose, $50 \mathrm{mg}$ of PCB-SS-PCL-SS-PCB was first resolved in the $4 \mathrm{ml}$ mixed solution of equivoluminal dimethyl sulfoxide (DMSO) and pure water. Subsequently, the solution was incubated with $0.5 \mathrm{ml} \mathrm{EDC} \cdot \mathrm{HCl}(400 \mathrm{mM})$ and NHS $(200 \mathrm{mM})$ in water for $30 \mathrm{~min}$ and then adjusted the $\mathrm{pH}$ value to 9 by adding in $10 \mathrm{mM}$ sodium borate buffer. After that, $5 \mathrm{mg}$ of GAL was added and reacted at room temperature (RT) for $4 \mathrm{~h}$. The solution was transferred into a dialysis bag (MWCO 3500) against pure water for $48 \mathrm{~h}$ and then lyophilized to obtain GAL-modified PCB-SSPCL-SS-PCB (GAL-m-polymer).

2.5. Preparation of GAL-M-Polymer Micelles. To prepare the GAL-m-polymer micelles (denoted as GAL-micelles), we used a similar procedure with that of PCB-SS-PCLSS-PCB micelles (denoted as PCB micelles) reported in our previous paper [28]. The size and size distribution (PDI) of GAL-micelles were measured with a laser particle size analyzer (Nano ZS90, Malvern, UK). The morphology of GAL-micelles was characterized with a JEM2100 (JEOL, Japan) transmission electron microscopy (TEM) system at an operated voltage of $75 \mathrm{kV}$. For TEM observation, a drop of micelle solution was added onto the copper grid, and then, the resulting sample was air-dried and measured at RT.

2.6. DOX Loading and Release from GAL-Micelles. We used a coprecipitation method to prepare DOX-loaded GAL-micelles. Briefly, $2 \mathrm{mg}$ DOX. $\mathrm{HCl}$ was first dissolved in DMSO and then treated by triethylamine solution $(1 \mathrm{mg} / \mathrm{ml})$ for $2 \mathrm{~h}$. Subsequently, $10 \mathrm{mg}$ GAL-m-polymer was added to the solution and stirred overnight. $\mathrm{PB}$ ( $50 \mathrm{mM}, \mathrm{pH} 7.4$ ) was added at a rate of one drop every 6-7 s until the micellization of GAL-m-polymer. The solution was sealed using a dialysis bag (MWCO 3500) against PB at $\mathrm{RT}$ for $48 \mathrm{~h}$, then filtered through a $0.45 \mu \mathrm{m}$ filter for removal of the free DOX. To calculate drug loading content, lyophilized DOX-loaded GAL-micelles were dissolved in DMSO and analyzed at $485 \mathrm{~nm}$ with a UV-Vis spectrophotometer
(UV2550, Shimadzu, Japan). Drug loading content (DLC) and drug loading efficiency (DLE) were determined by the following equations:

$$
\begin{aligned}
& \operatorname{DLC}(\%)=\frac{\text { weight of loaded drug }}{\text { weight of drug-loaded micelles }} \times 100 \% \\
& \operatorname{DLE}(\%)=\frac{\text { weight of loaded drug }}{\text { weight of drug in feed }} \times 100 \%
\end{aligned}
$$

In vitro release profiles DOX from GAL-micelles were studied using a dialysis tube (MWCO 14000 ) at $37^{\circ} \mathrm{C}$ against $\mathrm{PB}(\mathrm{pH} 5.0,50 \mathrm{mM})$ and $\mathrm{PB}(\mathrm{pH} 7.4,50 \mathrm{mM})$ in the presence and absence of $10 \mathrm{mM}$ DTT. At regular intervals, $3 \mathrm{ml}$ of release media was collected and an equal volume of fresh media was added. The amount of released DOX was determined by UV-Vis spectroscopy. The release experiments were conducted in triplicate. The results showed the average value with standard deviations.

2.7. Cell Uptake Studies. The cellular uptake of DOX, DOX-loaded PCB micelles, and DOX-loaded GAL-micelles was observed by fluorescence microscopy. HepG2 and EC109 cells were seeded into 24 -well plates with $5 \cdot 104$ cells/well in $1 \mathrm{ml}$ DMEM (10\% FBS) and incubated at $37^{\circ} \mathrm{C}$ in $5 \% \mathrm{CO}_{2}$. After reaching about $80 \%$ confluence, the cells were incubated with free DOX, DOX-loaded PCB micelles, or DOX-loaded GAL-micelles, where the concentration of DOX was fixed at $5 \mu \mathrm{g} / \mathrm{ml}$ in each culture medium. After $4 \mathrm{~h}$ incubation, the cells were washed three times with PBS and then imaged by fluorescence microscopy (IX73, Olympus, Japan). After that, the cells were treated with $0.25 \%$ trypsin and resuspended in $500 \mu \mathrm{l}$ PBS ( $\mathrm{pH} 7.4$ ) for flow cytometer (FC500, Beckman Coulter, US) measurement.

2.8. Cell Viability Assays. The cytotoxic effects of empty micelles or micelle-loading drug were determined using MTT assays. HepG2 and EC109 were seeded into 96-well plates at 5000 cells/well and cultured $24 \mathrm{~h}$. The culture medium was replaced with PBS ( $\mathrm{pH}$ 7.4) containing free DOX, DOX-loaded PCB micelles, or DOX-loaded GALmicelles, and then, the cells were incubated for another $24 \mathrm{~h}$. The medium was replaced with $200 \mu \mathrm{l}$ of fresh medium, and $20 \mu \mathrm{l} \mathrm{MTT}(5 \mathrm{mg} / \mathrm{ml}$ in PBS) was then added to each well. After $4 \mathrm{~h}$, unreacted dye was carefully removed and formazan crystals were dissolved in $200 \mu \mathrm{l} /$ well DMSO. The plate was incubated in $37^{\circ} \mathrm{C}$ for $10 \mathrm{~min}$ before measuring absorbance at $570 \mathrm{~nm}$ with an ELISA microplate reader (Bio-Rad). Cell viability (\%) was calculated as follows:

$$
\text { Cell viability }(\%)=\frac{\mathrm{OD}_{\text {sample }}}{\mathrm{OD}_{\text {control }}} \times 100,
$$

where $\mathrm{OD}_{\text {sample }}$ is the absorbance of the cells treated by polymers and $\mathrm{OD}_{\text {control }}$ is the absorbance of the untreated cells. Each experiment was done in triplicate.

2.9. In Vivo Antitumor Studies. The nude mice bearing HepG2 tumor model were injected via the tail vein with $200 \mu \mathrm{l}$ PBS, DOX, DOX-loaded PCB micelles, or DOX- 


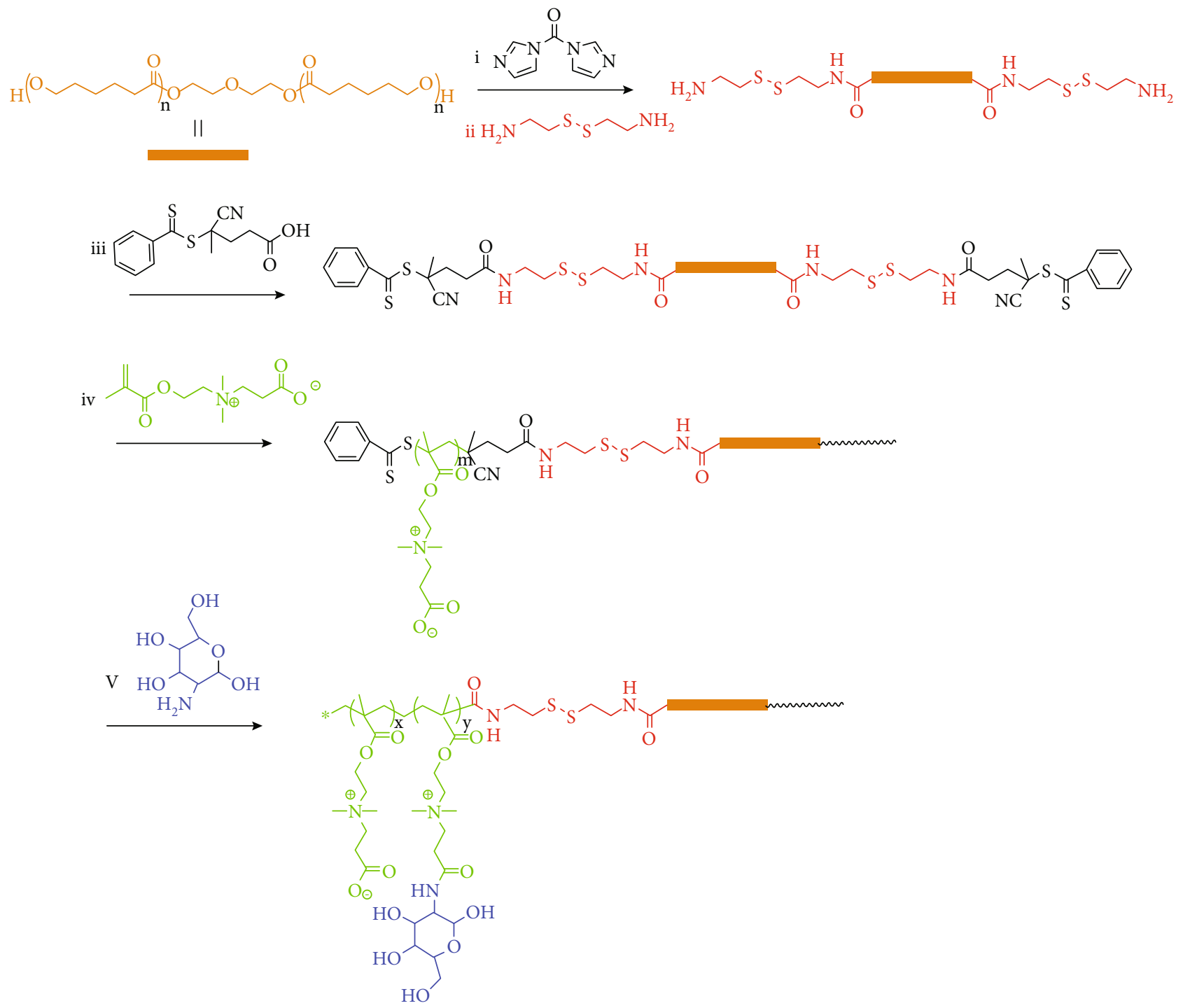

Scheme 2: Synthesis route of GAL-m-polymer. (i) CDI, anhydrous DCM, RT., 24 h; (ii) DMSO, pyridine, TEA, RT, 48 h; (iii) DCC, NHS, RT., 48 h; (iv) AIBN, THF, saturated salt water, $60^{\circ} \mathrm{C}, 48 \mathrm{~h}$; (V) EDC.HCl, NHS, DMSO, water, RT.

loaded GAL-micelles at an equivalent DOX dose of $4 \mathrm{mg} / \mathrm{kg}$ every two days. The tumor size and the body weight of mice were measured before each injection. Tumor size was measured using vernier calipers and calculated using the following formula: $V=d^{2} \times D / 2$ (where $d$ is width and $D$ is length, respectively). The therapeutic efficacy of the treatment was evaluated by comparing the experimental groups with the control group.

\section{Results and Discussion}

3.1. Polymer Characterization. Scheme 2 represents the synthesis route of GAL-m-polymer. PCL-diol was first introduced the cystamine at the end groups [31]. Next, a PCL macro-RAFT agent was readily obtained by a conjugating reaction of amino-end groups of PCL and the carboxylic group of CPADB. PCB-SS-PCL-SS-PCB was prepared by RAFT polymerization of CBMA by using CPADB-SS-PCLSS-CPADB as the chain transfer agent. Finally, GAL-m- polymer was prepared by modified PCB-SS-PCL-SS-PCB with GAL via EDC/NHS chemistry.

The resulting PCB-SS-PCL-SS-PCB and GAL-modified PCB-SS-PCL-SS-PCB (GAL-m-polymer) were characterized with ${ }^{1} \mathrm{HNMR}$ and FT-IR spectrum. ${ }^{1} \mathrm{HNMR}$ spectrum of GAL-m-polymer (Figure 1(a)) presents new small characteristic peaks at $1,2,3,4$, and $5(\delta 5.2,3.9,3.8,3.6$, and $3.4 \mathrm{ppm}$, respectively) which are attributed to the protons of GAL [30], in addition to the characteristic protons of PCB-SS-PCL-SSPCB [28]. FT-IR spectra were also used to determine the successful modification of PCB with GAL (Figure 1(b)). Compared with FT-IR spectrum of PCB-SS-PCL-SS-PCB, the absorption peak around $3400 \mathrm{~cm}-1$ broadening in that of GAL-m-polymer indicates the increased hydroxyl after the modification. Besides, the newly emerging peaks around $1656 \mathrm{~cm}^{-1}$ belongs to vibration of $\mathrm{C}=\mathrm{O}$ in the amide.

3.2. Characterization of GAL-m-Polymer Micelles. GAL-mpolymer was self-assembled into polymeric micelles in 


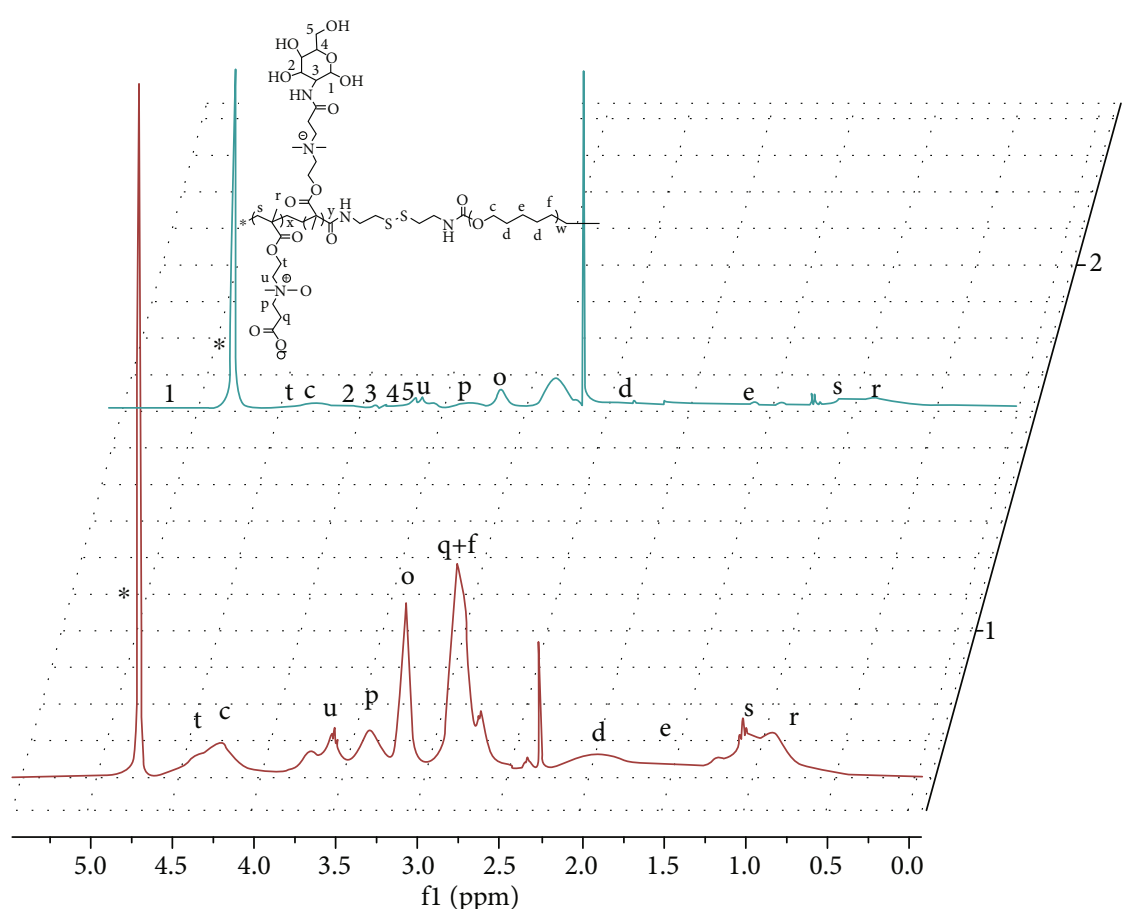

(a)

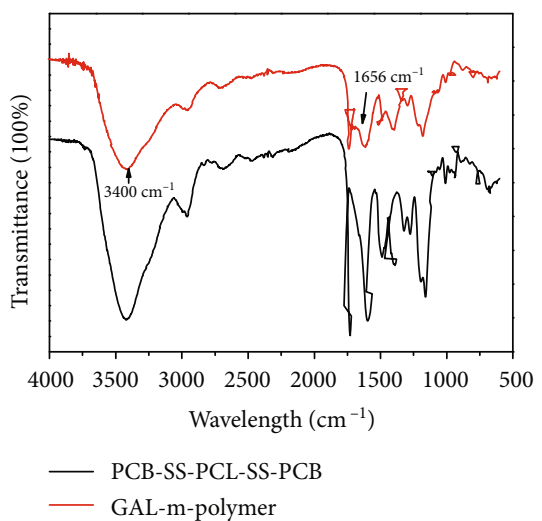

(b)

Figure 1: ${ }^{1}$ HNMR spectra (a) and FT-IR spectra (b) of PCB-SS-PCL-SS-PCB and GAL-M-polymer.

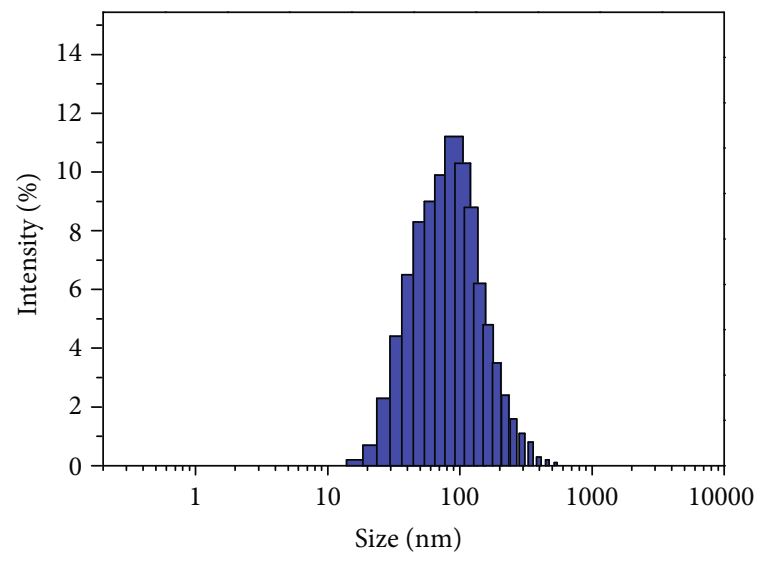

(a)

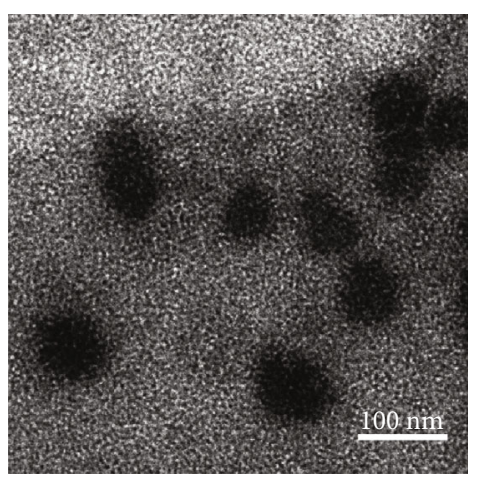

(b)

Figure 2: Hydrodynamic diameter distribution $f\left(D_{\mathrm{h}}\right)$ (a) and TEM image (b) of GAL-m-polymer micelles.

aqueous solution with PCL as a core and modified PCB as a shell. The hydrodynamic diameter distribution $\left(D_{h}\right)$ of micelle was measured by DLS (Figure 2(a)). The average diameter is approximately $91 \pm 4 \mathrm{~nm}$, showing a slight change compared to that of without modification [30]. The polydispersity index (PDI) is approximately 0.18 . TEM imaging revealed that the micelles of GAL-mpolymer have a spherical morphology with a mean diameter of approximately $75 \pm 3 \mathrm{~nm}$ (Figure 2(b)). The difference between DLS and TEM measurements of micellar size is likely due to the shape of micelle at different hydration states, from swelled at hydrated (DLS) to collapsed at dry (TEM).
The antifouling and redox-responsive properties of $\mathrm{PCB}$ micelles and GAL micelles were investigated by DLS analysis. After incubation with 50\% FBS solution, both micelles show no significant changes in size even after incubation for $72 \mathrm{~h}$ (Figure 3(a)), indicating that the zwitterionic PCB shell effectively prevents protein adsorption from polymer micelles. High serum stability is helpful to prolong circulation in the bloodstream. Reductive response to GSH was further investigated by analyzing size change with DLS. Both micelles show an increased $D_{h}$ and broadened PDI after addition of $10 \mathrm{mM}$ GSH for $12 \mathrm{~h}$. As disulfides are cleavable in the presence of GSH, the PCB shells are detached from the micellar surface, resulting in micelle aggregation. 


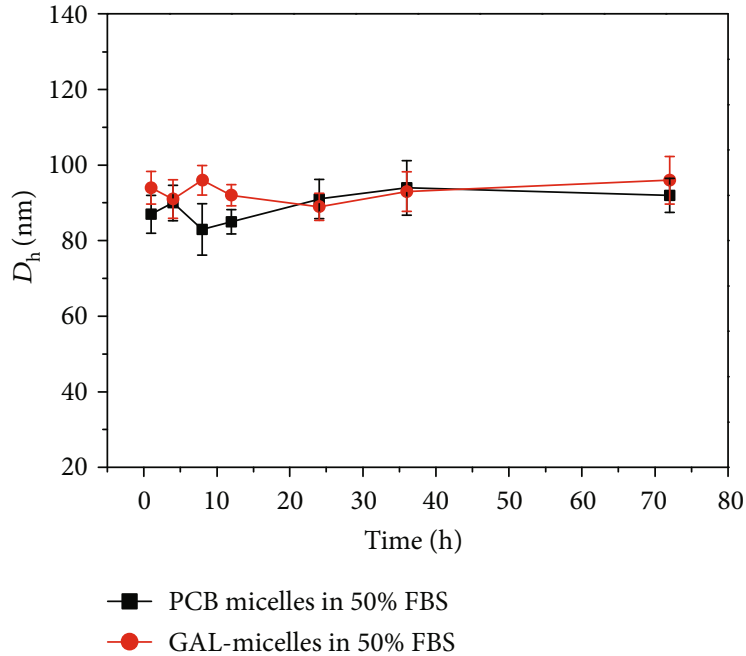

(a)

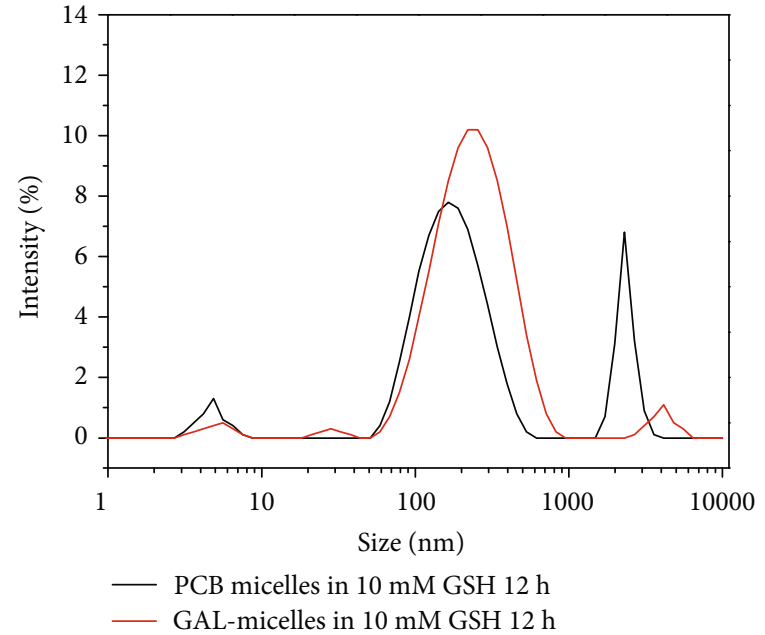

(b)

FIgure 3: Hydrodynamic diameter distribution $f\left(D_{\mathrm{h}}\right)$ of micelles and GAL-micelles in PB (50 mM, pH 7.4) containing 50\% FBS (a) and in PB $(50 \mathrm{mM}, \mathrm{pH}$ 7.4) containing $10 \mathrm{mM}$ GSH (b). Data are presented as the average (standard deviation $(n=3))$.

3.3. Preparation of DOX-Loaded GAL-Micelles. The multifunctional micellar carrier prepared by GAL-M-polymer is illustrated in Figure 4(a), where the PCB segment provided antifouling capability; the conjugated GAL ligand was expected an active targeting function; PCL was used for drug encapsulation and cystamine linkers offered a reductive response. Drug loading content was determined by UV-Vis spectrophotometry in DMSO. DLC and DLE were approximately $19.6 \%$ and $47.3 \%$, respectively. The average diameter of DOX-loaded GAL-micelles increased to $143 \pm 4 \mathrm{~nm}$ and also maintained a narrow size distribution (Figure 4(b)), when compared to GAL-micelles (Figure 2(b)). TEM image showed that DOX-loaded GAL-micelles have a spherical morphology with good dispersion and a diameter of approximately $167 \pm 7 \mathrm{~nm}$ (Figure $4(\mathrm{c})$ ). The results illustrate surface modification with GAL ligand show little influence on drug loading and carrier's properties [30] but may endow a novel function of active targeting to HepG2 cells. The stable structure and uniform small size facilitate intracellular uptake and EPR effect of carriers efficiently in vivo [32].

3.4. In Vitro Drug Release. In vitro drug release profile from DOX-loaded GAL-micelles was investigated at $37^{\circ} \mathrm{C}$ in $\mathrm{PBS}$ at pH 7.4 and 5.0 with or without $10 \mathrm{mM}$ GSH and as shown in Figure 5, only approximately 24\% of DOX was released from GAL-micelles within $48 \mathrm{~h}$ at $\mathrm{pH} 7.4$ without treated by GSH. As the increase of acidity to $\mathrm{pH} 5.0$, the DOX release reached to $48.2 \%$ within $24 \mathrm{~h}$, which is likely to result from the protonation of DOX. In comparison, an accelerated release of DOX was activated in the presence of $10 \mathrm{mM}$ GSH, in which over $60 \%$ and $80 \%$ of DOX was released from GAL-micelles at both $\mathrm{pH} 7.4$ and 5.0, respectively. The reductive response triggered a rapid drug release which is due to the cleavage of disulfide bonds at the surface of the core [33], leading to shell shedding and micelle aggregating, followed by fast drug efflux during the reassembly of deprotected micelles [34].
3.5. In Vitro Cytotoxicity and Cell Uptake. In vitro cytocompatibility of PCB micelles and GAL-micelles was evaluated with MTT assays in HepG2 and EC109 cells. Cells without treatment were used as control and showed a viability of $100 \%$. As shown in Figure 6(a), the viability of HepG2 and EC109 cell was over $90 \%$ incubation of both micelles, even at a concentration up to $200 \mathrm{mg} / \mathrm{l}$. The micelles possess low cytotoxicity which was likely due to the biocompatible PCB, PCL, and GAL ligand. For the cytotoxicity assays, HepG2 and EC109 cells were incubated with free DOX, DOXloaded PCB micelles, and DOX-loaded GAL-micelles (Figure 6(b)). The inhibitory concentrations to produce $50 \%$ of cell death (IC50) in HepG2 cells were $0.26 \mathrm{mg} / \mathrm{l}$, $1.44 \mathrm{mg} / \mathrm{l}$, and $0.72 \mathrm{mg} / \mathrm{l}$ for free DOX, DOX-loaded PCB micelles, and DOX-loaded GAL-micelles, respectively (Figure 6(b)). The DOX-loaded PCB micelles showed significantly lower cytotoxicity than free DOX, which may be due to less efficient cell uptake caused by the stealth shielding shell of zwitterionic PCB $[15,16]$. After the functionalization with GAL, DOX-loaded GAL-micelles exhibited increased inhibition of cellular proliferation when compared with DOX-loaded PCB micelles. However, DOX-loaded PCB micelles and DOX-loaded GAL-micelles showed similar antitumor activity in EC109 cells.

DOX is a popular chemotherapeutic drug and widely used in clinics for treating various hematological malignancies and solid tumors. Mechanistically, the insertion of DNA into tumor cells inhibits macromolecular biosynthesis, eventually leading to cell apoptosis [35, 36]. Intracellular drug accumulation depends on the efficacy of cell uptake and drug release from the loading carrier [37]. The increased therapeutic effect of DOX-loaded GAL-micelles in HepG2 cells can be attributed to improve cell uptake via active targeting, as demonstrated by fluorescence microscopy (Figure 6(c)). Indeed, empty micelles had no fluorescence signal, whereas the red fluorescence of DOX was easily detectable (Figure 6(c), B), showing that DOX is efficiently 


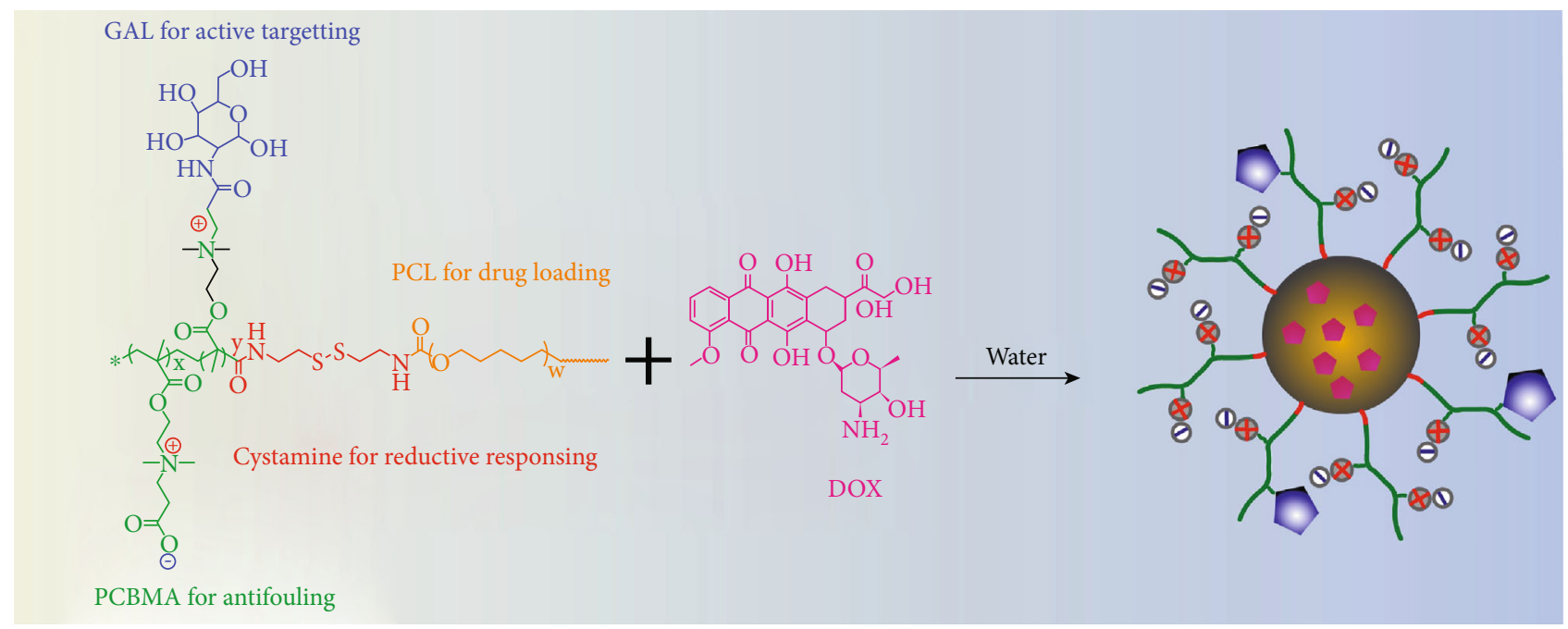

(a)

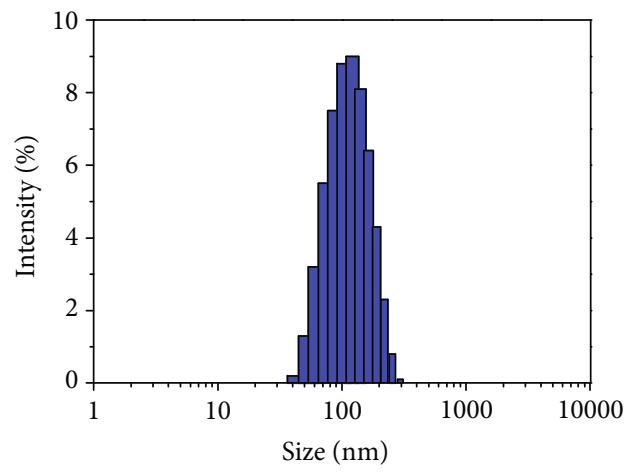

(b)

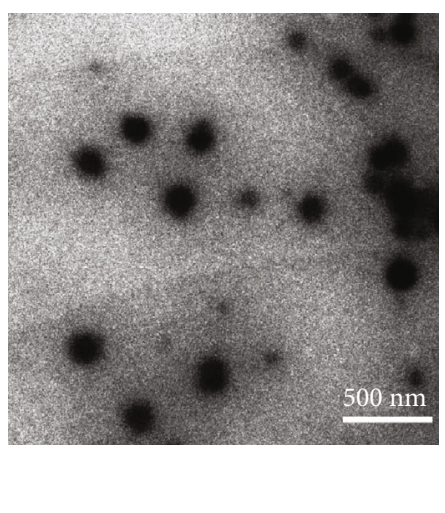

(c)

FIGURE 4: Schematic diagram of the preparation process of DOX-loaded GAL-micelles (a); hydrodynamic diameter distribution $f\left(D_{\mathrm{h}}\right)(\mathrm{b})$ and TEM image (c) of DOX-loaded GAL-micelles.

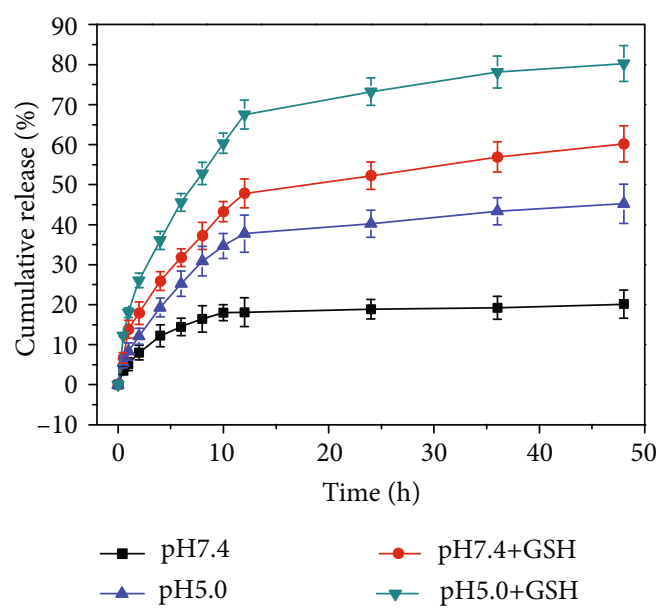

FIGURE 5: In vitro release profile of DOX-loaded GAL-micelles in PBS $(0.1 \mathrm{M})$ at $\mathrm{pH} 7.4$ and $\mathrm{pH} 5.0$ with or without GSH $(10 \mathrm{mg} / \mathrm{ml})$. Data are presented as the average (standard deviation $(n=3))$. released from DOX-loaded PCB micelles in response to intracellular GSH. Furthermore, DOX fluorescence in the cytoplasm and nucleus was brighter and more intense in DOX-loaded GAL-micelles (Figure 6(c), C), consistent with the increased cell uptake due to GAL binding to ASGP receptors on the surface of HepG2 cells [22]. However, DOX-loaded GAL-micelles showed a similar DOX fluorescence signal than DOX-loaded PCB micelles in EC109 cells, suggesting that active targeting of GAL is less effective in this cell type (Figure 6(d)). These results are consistent with the toxicity studies described above. Together, our data demonstrate that DOX-loaded GAL-micelles possess outside active targeting and inside redox-responsive release in HepG2 cells, suggesting they are promising nanocarriers for efficient treatment of liver cancer in vivo.

3.6. In Vivo Antitumor Efficacy. The antitumor efficacy of DOX-loaded micelles was investigated in vivo by using a nude mice bearing tumor model of human liver cancer. When the tumor size reached approximately $100 \mathrm{~mm}^{3}, \mathrm{PBS}$, free DOX, DOX-loaded PCB micelles, or DOX-loaded micelles GAL were injected into the tumor-bearing mice 


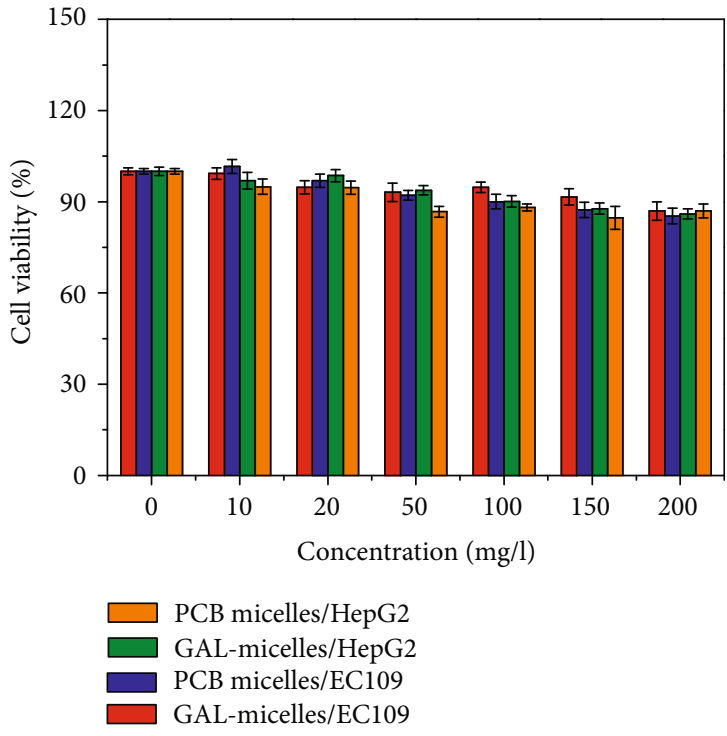

(a)
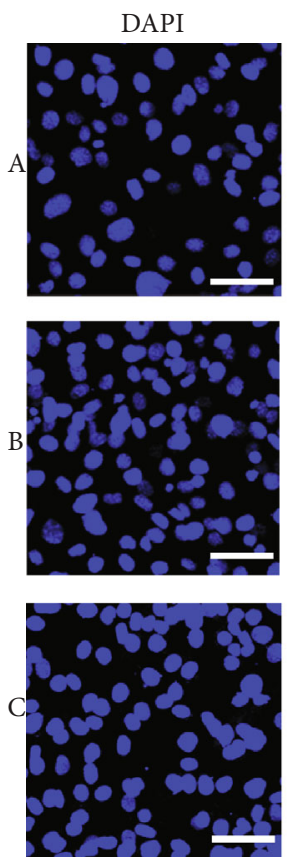

Merged
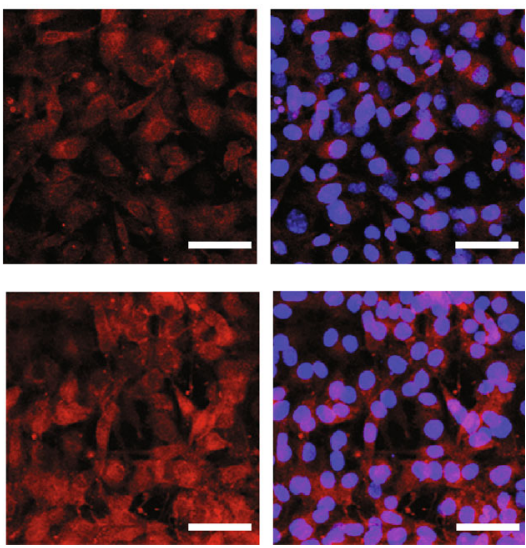

(c)

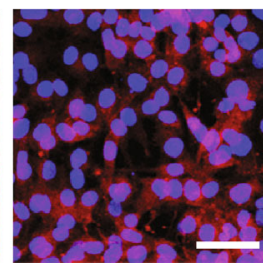

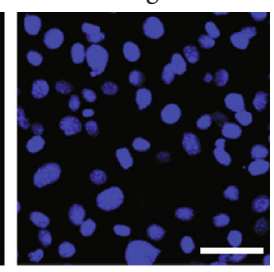

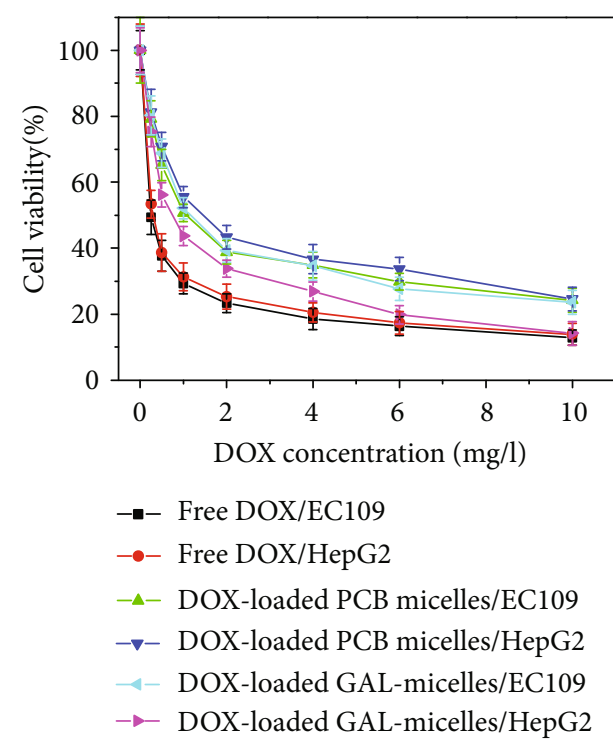

(b)
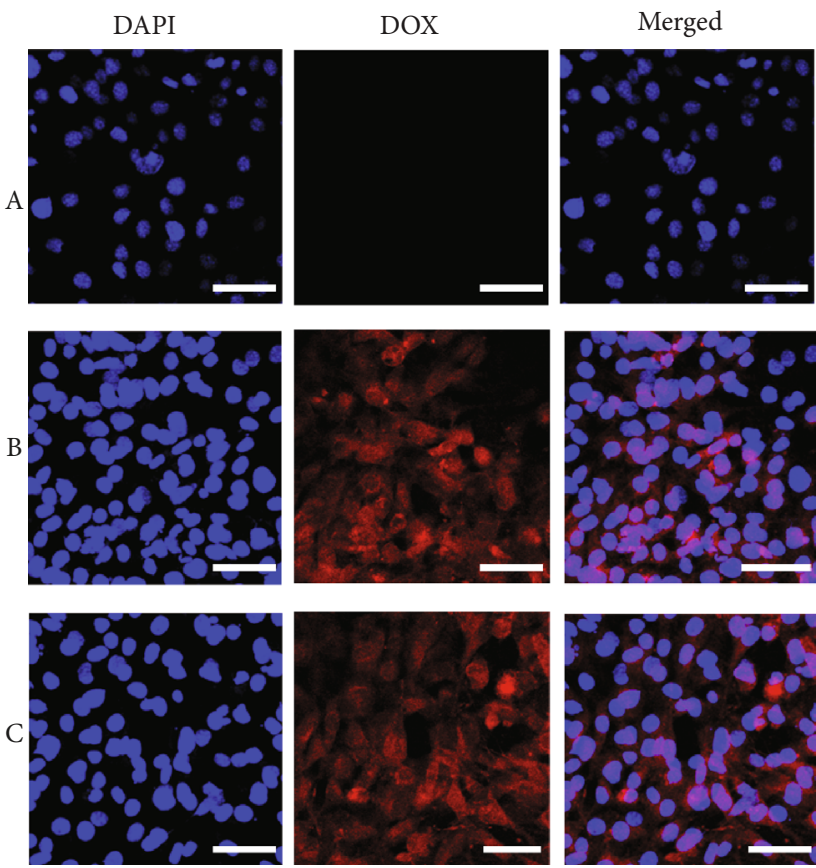

(d)

Figure 6: Toxicity of PCB micelles and GAL-micelles ( $a, b)$ and free DOX, DOX-loaded PCB-micelles, and DOX-loaded GAL micelles in HepG2 and EC109 cells incubated for $24 \mathrm{~h}$. Data are presented as the average (standard deviation $(n=3)$ ); fluorescence microscopy images of HepG2 (c) and EC109 cells (d) incubated with empty GAL-micelles (A), DOX-loaded PCB-micelles (B), and DOX-loaded GAL-micelles (C) for $4 \mathrm{~h}$, where the concentration of DOX was fixed at $5 \mu \mathrm{g} / \mathrm{ml}$. The scale bars correspond to $50 \mu \mathrm{m}$ in all the images.

through the tail vein. Tumor volume and body weight were measured and plotted as a function of time (Figure 7). As shown in Figure 7(a), the tumor volume in mice treated with PBS increased rapidly. In contrast, in the other groups, the tumors showed a slow growth, particularly, tumors in mice injected with DOX-loaded GAL-micelles. The body weight of mice treated with free DOX showed a clear decrease in weight as a result of in vivo cytotoxicity [21] (Figure 7(b)), the mice in the other groups exhibited a slow increase in body weight. More importantly, mice treated with DOX-loaded GAL-micelles had the smallest increase in weight within 12 days, suggesting the most effective tumor inhibition. 


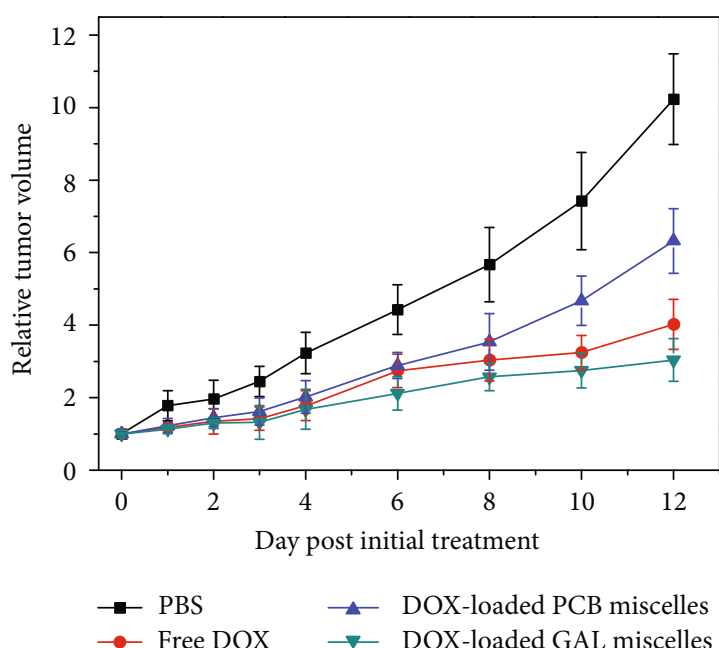

(a)

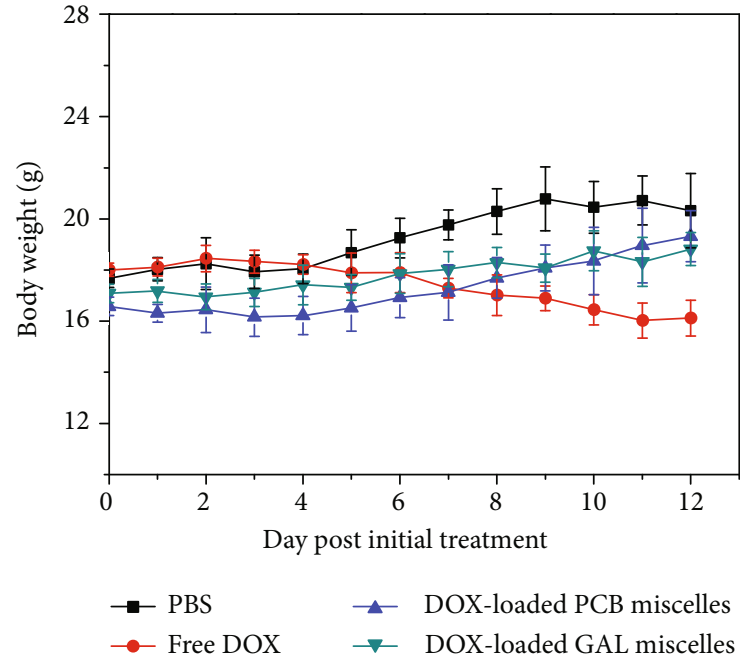

(b)

FIgURE 7: Tumor volume changes of the Bcap37 tumor-bearing nude mice after treatment with PBS or DOX at an equivalent dose of $4 \mathrm{mg} / \mathrm{kg}$ at each day (a). Changes in mice body weight during the treatment (b). Data are presented as the average (standard deviation, $n=3$ )).

Excellent stealth property from zwitterionic shells provides drug-loaded micelles a prolonged circulation time [38, 39] and enhanced drug accumulation at tumor site via EPR effect. The active targeting of DOX-loaded GAL-micelles to HepG2 cells further improves cell uptake. Finally, DOX is rapidly released due to reductive cleavage of disulfide bonds inside HepG2 cells.

\section{Conclusions}

The multifunctionality of polymeric micelle carrier, including high antifouling ability, redox responsive property, and active targeting in hepatic carcinoma cells, was successfully achieved by the modification of a triblock copolymer with disulfide-linked zwitterionic and biodegradable polymer (PCB-SS-PCL-SS-PCB) with galactosamine. GAL-micelles loaded with DOX exhibit rapid drug release under reductive environment, enhanced drug levels, and antitumor activity in HepG2 cells. As expected, this carrier revealed better anticancer efficacy in hepatic tumor-bearing mice than free DOX or no-targetable micelles. Thus, multifunctional nanocarriers with a flexible design of zwitterionic polymeric micelles represent a valuable strategy for improving drug bioavailability in the treatment of liver cancer.

\section{Data Availability}

The authors confirm that the data supporting the findings of this study are available within the article.

\section{Conflicts of Interest}

The authors declare no conflict of interest.

\section{Acknowledgments}

The authors gratefully acknowledge the financial support from the National Natural Science Foundation of China (Project U1704150) and Scientific and Technological Projects of Henan province (182102410017).

\section{References}

[1] S. Popat and M. O'Brien, "Chemotherapy strategies in the treatment of small cell lung cancer," Anti-Cancer Drugs, vol. 16, no. 4, pp. 361-372, 2005.

[2] C. Yang, M. Zhang, and D. Merlin, “Advances in plant-derived edible nanoparticle-based lipid nano-drug delivery systems as therapeutic nanomedicines," Journal of Materials Chemistry $B$, vol. 6, no. 9, pp. 1312-1321, 2018.

[3] K. Seidi, H. A. Neubauer, R. Moriggl, R. Jahanban-Esfahlan, and T. Javaheri, "Tumor target amplification: implications for nano drug delivery systems," Journal of Controlled Release, vol. 275, pp. 142-161, 2018.

[4] V. Sauraj, V. Kumar, B. Kumar et al., "Lipophilic 5-fluorouracil prodrug encapsulated xylan-stearic acid conjugates nanoparticles for colon cancer therapy," International Journal of Biological Macromolecules, vol. 128, pp. 204-213, 2019.

[5] F. Liu, Y. Sun, C. Kang, and H. Zhu, "Pegylated drug delivery systems: from design to biomedical applications," Nano Life, vol. 6, article 1642002, 2016.

[6] J. Pei, H. Hall, and N. D. Spencer, "The role of plasma proteins in cell adhesion to PEG surface-density-gradient- modified titanium oxide," Biomaterials, vol. 32, no. 34, pp. 8968-8978, 2011.

[7] C. Deng, Y. Jiang, R. Cheng, F. Meng, and Z. Zhong, "Biodegradable polymeric micelles for targeted and controlled anticancer drug delivery: promises, progress and prospects," Nano Today, vol. 7, no. 5, pp. 467-480, 2012.

[8] J. Qi, C. Sun, A. Zebibula et al., "Real-time and high-resolution bioimaging with bright aggregation-induced emission dots in 
short-wave infrared region," Advanced Materials, vol. 30, no. 12, article 1706856, 2018.

[9] C. E. Henry, Y.-Y. Wang, Q. Yang et al., “Anti-PEG antibodies alter the mobility and biodistribution of densely PEGylated nanoparticles in mucus," Acta Biomaterialia, vol. 43, pp. 61-70, 2016.

[10] J. J. F. Verhoef and T. J. Anchordoquy, "Questioning the use of PEGylation for drug delivery," Drug Delivery and Translational Research, vol. 3, no. 6, pp. 499-503, 2013.

[11] C. Leng, H. Hung, S. Sun et al., "Probing the surface hydration of nonfouling zwitterionic and PEG materials in contact with proteins," ACS Applied Materials \& Interfaces, vol. 7, no. 30, pp. 16881-16888, 2015.

[12] K. Seetho, S. Zhang, K. A. Pollack et al., "Facile synthesis of a phosphorylcholine-based zwitterionic amphiphilic copolymer for anti-biofouling coatings," ACS Macro Letters, vol. 4, no. 5, pp. 505-510, 2015.

[13] H. Sun, M. Y. Z. Chang, W.-I. Cheng et al., "Biodegradable zwitterionic sulfobetaine polymer and its conjugate with paclitaxel for sustained drug delivery," Acta Biomaterialia, vol. 64, pp. 290-300, 2017.

[14] Y. Men, S. Peng, P. Yang et al., "Biodegradable zwitterionic nanogels with long circulation for antitumor drug delivery," ACS Applied Materials \& Interfaces, vol. 10, no. 28, pp. 23509-23521, 2018.

[15] H. Ou, T. Cheng, Y. Zhang et al., "Surface-adaptive zwitterionic nanoparticles for prolonged blood circulation time and enhanced cellular uptake in tumor cells," Acta Biomaterialia, vol. 65, pp. 339-348, 2018.

[16] E. Muro, T. Pons, N. Lequeux et al., "Small and stable sulfobetaine zwitterionic quantum dots for functional live-cell imaging," Journal of the American Chemical Society, vol. 132, no. 13, pp. 4556-4557, 2010.

[17] S. Jiang and Z. Cao, "Ultralow-fouling, functionalizable, and hydrolyzable zwitterionic materials and their derivatives for biological applications," Advanced Materials, vol. 22, no. 9, pp. 920-932, 2010.

[18] Z. Zhang, S. Chen, and S. Jiang, "Dual-functional biomimetic materials: nonfouling poly(carboxybetaine) with active functional groups for protein immobilization," Biomacromolecules, vol. 7, no. 12, pp. 3311-3315, 2006.

[19] H. Vaisocherová, W. Yang, Z. Zhang et al., "Ultralow fouling and functionalizable surface chemistry based on a zwitterionic polymer enabling sensitive and specific protein detection in undiluted blood plasma," Analytical Chemistry, vol. 80, no. 20, pp. 7894-7901, 2008.

[20] A. J. Keefe and S. Jiang, "Poly(zwitterionic)protein conjugates offer increased stability without sacrificing binding affinity or bioactivity," Nature Chemistry, vol. 4, no. 1, pp. 59-63, 2012.

[21] W. Lin, G. Ma, N. Kampf, Z. Yuan, and S. Chen, "Development of long-circulating zwitterionic cross-linked micelles for active-targeted drug delivery," Biomacromolecules, vol. 17, no. 6, pp. 2010-2018, 2016.

[22] D. Zhu, W. Tao, H. Zhang et al., "Docetaxel (DTX)-loaded polydopamine-modified TPGS-PLA nanoparticles as a targeted drug delivery system for the treatment of liver cancer," Acta Biomaterialia, vol. 30, pp. 144-154, 2016.

[23] H.-F. Liang, C.-T. Chen, S.-C. Chen et al., "Paclitaxel-loaded $\operatorname{poly}(\gamma$-glutamic acid)-poly(lactide) nanoparticles as a targeted drug delivery system for the treatment of liver cancer," Biomaterials, vol. 27, no. 9, pp. 2051-2059, 2006.
[24] H.-W. Chien, P.-H. Cheng, S.-Y. Chen, J. Yu, and W.-B. Tsai, "Low-fouling and functional poly(carboxybetaine) coating via a photo-crosslinking process," Biomaterials Science, vol. 5, no. 3, pp. 523-531, 2017.

[25] Z. Cao, Q. Yu, H. Xue, G. Cheng, and S. Jiang, "Nanoparticles for drug delivery prepared from amphiphilic PLGA zwitterionic block copolymers with sharp contrast in polarity between two blocks," Angewandte Chemie International Edition, vol. 49, no. 22, pp. 3771-3776, 2010.

[26] Z. Cao, Y. Ma, C. Sun et al., "ROS-sensitive polymeric nanocarriers with red light-activated size shrinkage for remotely controlled drug release," Chemistry of Materials, vol. 30, no. 2, pp. 517-525, 2018.

[27] N. Rapoport, R. Gupta, Y. S. Kim, and B. E. O'Neill, “Polymeric micelles and nanoemulsions as tumor-targeted drug carriers: insight through intravital imaging," Journal of Controlled Release, vol. 206, pp. 153-160, 2015.

[28] Y.-w. Hu, Y.-z. Du, N. Liu et al., "Selective redox-responsive drug release in tumor cells mediated by chitosan based glycolipid-like nanocarrier," Journal of Controlled Release, vol. 206, pp. 91-100, 2015.

[29] Sauraj, S. U. Kumar, V. Kumar, R. Priyadarshi, P. Gopinath, and Y. S. Negi, "pH-responsive prodrug nanoparticles based on xylan-curcumin conjugate for the efficient delivery of curcumin in cancer therapy," Carbohydrate Polymers, vol. 188, pp. 252-259, 2018.

[30] J. Jiang, J. Li, B. Zhou et al., "Fabrication of polymer micelles with zwitterionic shell and biodegradable core for reductively responsive release of doxorubicin," Polymers, vol. 11, no. 6, p. 1019, 2019.

[31] P. Davoodi, M. P. Srinivasan, and C.-H. Wang, "Synthesis of intracellular reduction-sensitive amphiphilic polyethyleneimine and poly( $\varepsilon$-caprolactone) graft copolymer for ondemand release of doxorubicin and p53 plasmid DNA," Acta Biomaterialia, vol. 39, pp. 79-93, 2016.

[32] Y. Chen, W. Zhang, Y. Huang, F. Gao, and X. Fang, "In vivo biodistribution and anti-tumor efficacy evaluation of doxorubicin and paclitaxel-loaded pluronic micelles decorated with c(RGDyK) peptide," PLoS One, vol. 11, no. 3, article e0149952, 2016.

[33] Sauraj, Vinay kumar, B. Kumar et al., "Redox responsive xylan-SS-curcumin prodrug nanoparticles for dual drug delivery in cancer therapy," Materials Science and Engineering: C, vol. 107, article 110356, 2020.

[34] H. Sun, B. Guo, X. Li et al., "Shell-sheddable micelles based on dextran-SS-poly( $\varepsilon$-caprolactone) diblock copolymer for efficient intracellular release of doxorubicin," Biomacromolecules, vol. 11, no. 4, pp. 848-854, 2010.

[35] X. Zhang, A. Poniewierski, K. Sozański, Y. Zhou, A. Brzozowska-Elliott, and R. Holyst, "Fluorescence correlation spectroscopy for multiple-site equilibrium binding: a case of doxorubicin-DNA interaction," Physical Chemistry Chemical Physics, vol. 21, no. 3, pp. 1572-1577, 2019.

[36] S. Wang, E. A. Konorev, S. Kotamraju, J. Joseph, S. Kalivendi, and B. Kalyanaraman, "Doxorubicin induces apoptosis in normal and tumor cells via distinctly different Mechanisms," Journal of Biological Chemistry, vol. 279, no. 24, pp. 25535-25543, 2004.

[37] H. Wei, R.-X. Zhuo, and X.-Z. Zhang, "Design and development of polymeric micelles with cleavable links for intracellular drug delivery," Progress in Polymer Science, vol. 38, no. 3-4, pp. 503-535, 2013. 
[38] Z. Wang, G. Ma, J. Zhang et al., "Development of zwitterionic polymer-based doxorubicin conjugates: tuning the surface charge to prolong the circulation and reduce toxicity," Langmuir, vol. 30, no. 13, pp. 3764-3774, 2014.

[39] W. Yang, S. Liu, T. Bai et al., "Poly(carboxybetaine) nanomaterials enable long circulation and prevent polymer-specific antibody production," Nano Today, vol. 9, no. 1, pp. 10-16, 2014. 